\title{
The importance of behavioral outcomes
}

John Hulland ${ }^{1} \cdot$ Mark Houston $^{2}$

Published online: 6 January 2021

(C) Academy of Marketing Science 2021
One of the most important tasks that we complete regularly as editors - often on a daily basis - is the initial assessment of every research paper submitted to JAMS. Based on our initial read of a manuscript, we either send the paper out to reviewers for evaluation or desk reject the work. One of the major frustrations that we far too frequently encounter in our initial review is the authors' sole use of intentions as the dependent variable(s) in their empirical research. Use of intention measures alone often undermines the potential contribution of the work, resulting in early rejection. There are two key reasons for this.

First, research has not found the relationship between intention and behavior to be a consistently strong one. For example, an early meta-analysis by Sheeran (2002) showed that "intentions explain only $28 \%$ of the variance in behavior." Whereas this may be significant and acceptable in some cases, substantial variability has been observed across research studies. Similarly, models estimating new product sales explicitly incorporate a gap between consumers' stated purchase intentions and observed sales (e.g., Morrison 1979; Silk and Urban 1978). ${ }^{1}$

This potentially weak connection is often referred to as the "intention-behavior gap," and it is driven in large part by individuals who indicate an intention to complete a behavior but then do not follow through. More recent reviews (e.g., Morwitz and Munz 2020; Sheeran and Webb 2016) have suggested that whereas correlations between intentions and behaviors represent, on average, "medium effect sizes," substantial variances in these effect sizes have been noted.

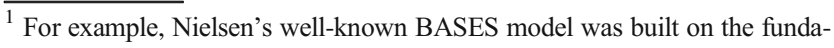
mental assumption that "consumers do not usually do what they claim to do, and often the difference between claims and behaviors is very significant" (https://www.ashokcharan.com/Marketing-Analytics/ ppv-BASES.php).

John Hulland

jhulland@uga.edu

1 Department of Marketing, Terry College of Business, University of Georgia, Athens, GA, USA

2 Department of Marketing, Neeley School of Business, Texas Christian University, Fort Worth, TX, USA
}

Summarizing, although intentions often relate to behaviors to a degree, the connection between the two is not invariably strong. Yet researchers often assume that the intentionbehavior gap does not exist.

Second, JAMS emphasizes, as a key part of its mission, the publication of managerially relevant research. What this means is that the practical implications of research submitted to the journal need to be both clear and actionable. Managers are interested in understanding how potential marketing interventions affect meaningful outcomes such as purchase, word-ofmouth generation, charitable cause donation, or other behavioral responses. These relevant findings can positively inform business performance, society, and the economy. Although attitudes and intentions may have changed because of the introduction of some other construct, these managers - if they are to invest in actions based on the research - need to see empirical evidence clearly demonstrating that the intervention actually influences the focal outcome behavior of interest.

We believe that two related reasons often drive a scholar's decision to use intention as an outcome. First, the researcher's primary interest may be in understanding the theoretical mechanisms (attitudes, intentions) that drive behavior rather than in the behavior itself. Such research clearly has value. For example, considerable early work using the theory of planned behavior focused on distinguishing beliefs and attitudes from intentions (Morwitz and Munz 2020). In these cases, "enhancing realism and utilizing behavioral measures isn't always necessary or even preferred" (Morales et al. 2017, p. 467). And we recognize that the precision required to craft and test a rich theoretical model to isolate these intervening variables is challenging enough without adding the complexity of measuring often-messy behavior in a valid way. Still, although we believe such research is valuable to our field at large, it often has a poor fit with JAMS.

This connects to the second reason scholars might measure intentions only: lab-based experiments focused on intentions can often be run more quickly and efficiently compared to when behavioral outcomes are assessed. In particular, subjects are typically more readily accessible due to the presence of student subject pools, behavioral labs, etc. 
However, there are many important contexts (both in marketing and elsewhere) where the intention-behavior gap is substantial, and where behavioral outcomes are needed, despite scholars' practical reasons for not wanting to gather them. In response to this conundrum, the goal of this editorial is to describe the intention-behavior gap problem, to offer a framework that may help to identify circumstances when the gap may be large (or small), and to suggest potential remedies to the problem where it exists. Although this is an issue most often encountered in consumer behavior research, it can also be problematic for managerial or strategy studies that rely on responses collected from individuals.

\section{Potential moderators}

The magnitude of the intention-behavior gap can be influenced by many different moderators. For example, Kalwani and Silk (1982, p. 278) observe that "the relationship between expressed intentions and subsequent purchase behavior appears to be different for generic durables as compared to branded package goods." In the retail setting, Seiders et al. (2005, p. 37) find that "customers often fail to consider intervening contingency effects when they predict their own future behavior." Carrington et al. (2014) cite research revealing that social desirability bias can often play a role in inflating individuals' stated intentions. Similarly, use of behavioral selfreports (versus objective measures) typically widens the gap. Although it is beyond the scope of this editorial to discuss these potential moderators in any detail, Webb and Sheeran (2006) suggest that they broadly fall into three groups. Conceptual moderators (e.g., volitional control) are variables that can be used to theoretically predict how strongly intentions lead to behavior. Measurement-based moderators can also play a role. For example, as the time interval between the measurement of an intention and a behavior increases, the intention-behavior gap typically grows (e.g., due to new information). Finally, study characteristics (e.g., type of sample) have been found to make a difference. For example, students (versus non-students) tend to answer questions both more readily and more consistently, resulting in smaller intention-behavior gaps.

Although we (and our readers at JAMS) have a strong preference for behavioral outcomes, authors of successful papers that rely on intention DVs alone compellingly articulate why the intention-behavior gap is likely to be negligible (versus substantial) in their work. Clearly, it is neither practical nor necessary to account for all of these potential moderators, but scholars should provide strong theoretical explanations as to why the gap can be expected to be small. Furthermore, they should carefully examine the consequences of their chosen methodology, to ensure it does not inflate the gap.

\section{An intentions realization framework}

One useful way to think about the extent to which intentions are realized in the form of behaviors is to consider the broad self-regulatory tasks (and challenges) that individuals encounter in their attempts to enact their intentions. As shown in Fig. 1, Sheeran and Webb (2016) detail various selfregulatory problems that an individual may face during distinct stages of goal-pursuit. They suggest (p. 506) that these problems include "(a) getting started, (b) keeping ongoing goal pursuit on track, and (c) bringing goal pursuit to a successful close."

For the behaviors of interest to JAMS, this framework suggests reasons why a consumer may ultimately fail to engage in a behavior despite having formed an intention to do so. For example, even if the consumer starts toward the goal of healthy eating, they can become distracted by competing opportunities (e.g., socializing with friends who regularly meet at a fast-food restaurant) or face resource allocation decisions (e.g., when seeing high prices for natural food options at the grocery store) that reveal low commitment to the initial intention.

\section{Potential remedies}

Although a range of options may be available to researchers wanting to address concerns regarding the potential for an intention-behavior gap, we describe below four distinct approaches: (1) explicit measurement of behavioral outcomes, (2) use of mixed methods to obtaining triangulating evidence, (3) use of "implementation intentions," and (4) creative measurement of "proxy" behaviors. (Although we describe these approaches individually, they can be used in conjunction with one another.)

\section{Explicit measurement of behavioral outcomes}

The most direct and obvious way to link intentions (as well as other constructs) to behavior is to directly measure the outcome behavior(s) of interest. Agreeing with Morales, Amir, and Lee $(2017$, p. 466), we define behavior as "actions, consciously intended or not, that [individuals] engage in." Although outcome behaviors in research studies are often conscious choices (e.g., decision to buy, what information to search, where to shop), they can also include unconscious physical manifestations (e.g., heart rate, voice intonations, facial expressions) as well as other relevant measures.

Whereas it may be easier to observe some of these outcome behaviors in the lab setting (e.g., pulse, consumption of food),

\footnotetext{
${ }^{2}$ Carrington et al. (2014) propose a somewhat similar approach in the specific context of ethical consumerism.
} 

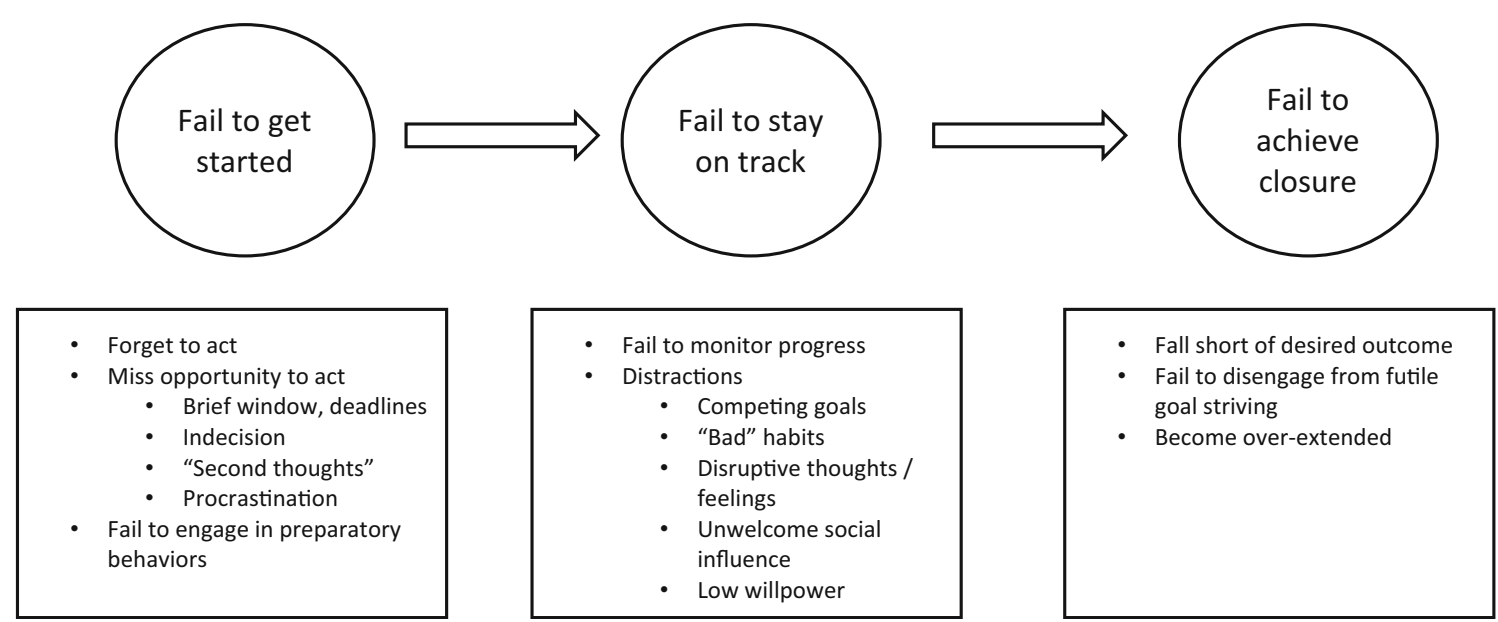

Fig. 1 Steps involved in failing to realize intentions. Note: Adapted from Sheeran and Webb (2016)

measuring behaviors in other contexts can prove challenging (e.g., field experiments, observing in-store purchases). It is generally not necessary to demonstrate a strong connection between intentions and behaviors in every study reported in a paper. Instead, one well-designed study that connects the two and convincingly demonstrates a strong interrelationship may be sufficient.

\section{Mixed methods}

One particularly effective way of accomplishing this goal is making use of mixed methods. In particular, field studies can play a powerful role in demonstrating a clear connection between focal antecedent constructs and outcome behaviors. ${ }^{3}$ (This is a primary reason for the substantial increase in published field-based research over the last decade.) A prototypical paper might use a single field study to show a causal connection between the focal construct and outcome behavior(s), and then several experimental studies to explicate (and test) the underlying mediating process (or processes). ${ }^{4}$ When this evidence is convergent, it strengthens the researcher's conclusions regarding the size of the intention-behavior gap.

\section{"Implementation intentions"}

The model shown in Fig. 1 indicates how individuals may fail in translating their intentions into behaviors. To overcome these challenges - and reduce the size of the intentionbehavior gap-Fennis et al. (2011, p. 303) suggest the use

\footnotetext{
$\overline{3}$ Field experiments and other types of field studies (e.g., "field data") can be quite different. For example, Morales et al. (2017) do an excellent job of distinguishing between field experiments (where subjects are unaware that a research study is taking place) and experiments in the field. However, whereas the methodological details are distinct, our recommendation to use behaviors rather than intentions applies to both.

${ }^{4}$ Field-based demonstrations are usually not expected to rule out alternative explanations or reveal underlying processes. Instead, they show a connection between the IVs and the DV(s) in a more realistic setting.
}

of "implementation intentions, or plans concerning where, when and how one will perform the intended behavior." Whereas explicitly instructing a person to form an intention to implement a behavior tends to produce reactance against doing so, Fennis et al. (2011, p. 304) suggest encouraging participants to visualize "specific situations" in which they might carry out the intended behavior and "imagine doing so" (e.g., when I walk by the natural food market on the way home from work, I'll stop in and stock up on fruits and vegetables). Ideally, this implementation will focus on creating situation-specific intentions that will reduce the attitudebehavior gap (i.e., situation-specific attitudes are connected to situation-specific behaviors). Study stimuli can also be used to present a more realistic context (e.g., an online store that carries many products; a store lab that is similar in appearance to the everyday shopping environment). In addition to the potential use of these if-then plans, Sheeran and Webb (2016) suggest that progress-monitoring interventions that encourage participants to reflect on the degree to which they are achieving intended goals can also be effective.

In the context of research submitted to JAMS, scholars can utilize this information in two ways. First, researchers can directly incorporate implementation interventions into their studies, strengthening the connection between intentions and behaviors. ${ }^{5}$ Second, where such interventions are not feasible, the model in Fig. 1 can still be used to identify — and thereby develop conceptual counter-arguments to try to ameliorateconcerns regarding the intention-behavior gap.

\section{Use "proxy" behaviors}

Under circumstances where focal behaviors of interest are difficult (or even impossible) to measure directly, use of proximal measures may be appropriate. Because such measures

\footnotetext{
${ }^{5}$ Fennis et al. (2011) suggest that use of "indirect" persuasive approaches may be appropriate in some consumer behavior contexts.
} 
may be indirect, triangulation obtained through multiple operationalizations of behaviors is a powerful way to present more compelling evidence. For example, Kristofferson et al. (2017) use a variety of different dependent measuresincluding the number of shots fired in a video game, physical response to a jammed vending machine, and testosterone level (collected using oral swabs) - to convincingly demonstrate that exposure to limited-quantity promotions can induce consumer aggression.

Recent advances in measurement technologies create many potential opportunities for scholars to creatively assess behavioral outcomes, depending on the objectives of the research. In addition to traditional behavioral outcomes such as product purchase and choice, researchers can also examine outcomes such as electronic word-of-mouth generation, purchase prices, amounts donated online to charitable causes, eye tracking and various other biometrics (e.g. heart rate, brain waves), and purchase quantities. A key issue in using such proxies is that they need to be relatively closely related to the behavioral outcome of interest. Ideally, evidence already exists that links these proxies to the target behavior.

\section{Conclusion}

Sheeran and Webb (2016, p. 511) conclude that the "intention-behavior gap is large - current evidence suggests that intentions get translated into action approximately one-half of the time." What this means for marketing researchers is that it is often inappropriate to design and conduct research studies that employ intentions as the ultimate dependent outcomes. Whenever possible, we encourage scholars to also examine behavioral outcomes that both strengthen the theoretical contributions of their research as well as offer important managerial insights.

We hope this editorial clarifies that field studies are not the only solution; use of one or more of the potential remedies we discuss can help to show that the intention-behavior gap is not large. When use of such measures is not feasible, we suggest active consideration of the various self-regulatory problems that might arise as an individual attempts to translate intentions into behaviors (Fig. 1).

Acknowledgments We thank the following people for their helpful feedback on earlier versions of this editorial: Marcus Cunha, O.C. Ferrell, Kelly Haws, Charlotte Mason, Stephanie Noble, Maura Scott, and Sarah Whitley.

\section{References}

Carrington, M. J., Neville, B. A., \& Whitwell, G. J. (2014). Lost in translation: Exploring the ethical consumer intention-behavior gap. Journal of Business Research, 67(1), 2759-2767.

Fennis, B. M., Adriaanse, M. A., Stroebe, W., \& Pol, B. (2011). Bridging the intention-behavior gap: Inducing implementation intentions through persuasive appeals. Journal of Consumer Psychology, 21(3), 302-311.

Kalwani, M. U., \& Silk, A. J. (1982). On the reliability and predictive validity of purchase intention measures. Marketing Science, 1(3), 243-286.

Kristofferson, K., McFerran, B., Morales, A. C., \& Dahl, D. W. (2017), The dark side of scarcity promotions: How exposure to limitedquantity promotions can induce aggression. Journal of Consumer Research, 43(5), 683-706.

Morales, A. C., Amir, O., \& Lee, L. (2017). Keeping it real in experimental research-Understanding when, where, and how to enhance realism and measure consumer behavior. Journal of Consumer Research, 44(2), 465-476.

Morrison, D. G. (1979). Purchase intentions and purchase behavior. Journal of Marketing, 43(2), 65-74.

Morwitz, V. G., \& Munz, K. P. (2020). Intentions. Consumer Psychology Review. https://doi.org/10.1002/arcp.1061.

Seiders, K., Voss, G. B., Grewal, D., \& Godfrey, A. L. (2005). Do satisfied customers buy more? Examining moderating influences in a retailing context. Journal of Marketing, 69(Oct.), 26-43.

Sheeran, P. (2002). Intention-Behavior relations: A conceptual and empirical review. European Review of Social Psychology, 12(1), 1-36.

Sheeran, P., \& Webb, T. L. (2016). The intention-behavior gap. Social and Personality Psychology Compass, 10(9), 503-518.

Silk, A. J., \& Urban, G. L. (1978). Pre-test-market evaluation of new packaged goods: A model and measurement methodology. Journal of Marketing Research, 15(2), 171-191.

Webb, T. L., \& Sheeran, P. (2006). Does changing behavioral intentions engender behavior change? A meta-analysis of the experimental evidence. Psychological Bulletin, 132(2), 249-268.

Publisher's note Springer Nature remains neutral with regard to jurisdictional claims in published maps and institutional affiliations. 